\title{
A CAE approach for the stress analysis of gear models by 3D digital photoelasticity
}

\author{
Paola Forte, Alessandro Paoli* and Armando Viviano Razionale \\ University of Pisa, Department of Civil and Industrial Engineering, Largo Lucio \\ Lazzarino, n.1 - 56126 Pisa, Italy \\ E-mail: p.forte@ing.unipi.it, a.paoli@ing.unipi.it and a.razionale@ing.unipi.it \\ - Author to whom correspondence should be addressed; E-Mail: \\ a.paoli@ing.unipi.it; Tel.: +39-050-221-8019; Fax: +39-050-221-8065.
}

\begin{abstract}
The use of numerical and experimental methods to determine the stress field of mechanical components is well known. In particular, 3D photoelasticity can be considered the only experimental technique for the complete stress state evaluation of $3 \mathrm{D}$ components. The advent of rapid prototyping techniques has allowed the manufacturing of complex models in a matter of hours by using birifrangent materials. The present paper is focused on the description of a Computer Aided Engineering (CAE) approach which combines Finite Element (FE) simulations and automatic photoelastic investigations for the stress analysis of face gear drives, made by stereolithography. Computer Aided Design (CAD) geometries, used to manufacture the stereolithographic models, are directly used to perform FE analyses, thus allowing the stress analysis process to become simpler and easier. The substantial agreement observed between experimental and numerical results proved the potentialities of the adopted approach and the usefulness of FE simulations to optimize photoelastic analyses through cost- and time-effective experiments even for complex 3D shapes.
\end{abstract}

Keywords: 3D photoelasticity, stereolithography, face gear models, FEA.

The final publication is available at Springer via http://dx.doi.org/ 10.1007/s12008013-0201-4 


\begin{abstract}
The use of numerical and experimental methods to determine the stress field of mechanical components is well known. In particular, 3D photoelasticity can be considered the only experimental technique for the complete stress state evaluation of 3D components. The advent of rapid prototyping techniques has allowed the manufacturing of complex models in a matter of hours by using birifrangent materials. The present paper is focused on the description of a Computer Aided Engineering (CAE) approach which combines Finite Element (FE) simulations and automatic photoelastic investigations for the stress analysis of face gear drives, made by stereolithography. Computer Aided Design (CAD) geometries, used to manufacture the stereolithographic models, are directly used to perform FE analyses, thus allowing the stress analysis process to become simpler and easier. The substantial agreement observed between experimental and numerical results proved the potentialities of the adopted approach and the usefulness of FE simulations to optimize photoelastic analyses through cost- and time-effective experiments even for complex 3D shapes.
\end{abstract}

Keywords: 3D photoelasticity, stereolithography, face gear models, FEA.

\title{
1. Introduction
}

Photoelasticity is a full-field technique which directly provides the information of principal stress difference and the orientation of principal stress direction by fringe analysis of components made of birifrangent materials [1]. Nowadays, numerical techniques, such as the finite element method (FEM), have progressively replaced experimental stress analysis during prototype design. However, photoelasticity may still play an important role in supporting numerical methods with the aim of increasing confidence when complex analyses must be carried out. In particular, three-dimensional (3D) photoelasticity can be considered the only technique for the experimental assessment of the complete stress state throughout complex mechanical components.

3D photoelasticity typically presents two sequential phases: the preparation of the models to be used for the experimental tests and the fringe analysis step. The advent of digital image processing techniques (digital photoelasticity) [2] has allowed photoelastic analyses to become faster and more accurate. On the other hand, 
the manufacturing and slicing of complex 3D photoelastic models still represents a troublesome task. In blocks using thermo-setting resins. These processes are expensive and time-consuming, with models typically requiring days to be completed.

In the last decades, rapid prototyping (RP) techniques have provided additive manufacturing processes, such as stereolitography, which allow 3D components to be built in a matter of hours [3]. Recently, some photoelasticians have analyzed the optical and mechanical properties of stereolitographic resins (usually acrylate- and epoxy-based resins) [4,5]. These studies have been mainly focused on the investigation of the influence of processing parameters, such as position and orientation of specimens in the resin bath, post curing treatments, build style, and on the material shrinkage as well as mechanical and birifrangent properties. The results published in the literature have evidenced that an interesting scenario can be opened within the full-field stress analysis of complex models. In this context, few researches have aimed at analyzing the stress distribution in three-dimensional stereolitographic complex specimens [6,7]. In particular, when experimental analyses concerning 3D photoelasticity are carried out, one of the most challenging issues relies on the determination of the most suitable load to be used for the stress freezing process. Large values of the applied load may improperly distort the model while too low values may result in a poor optical response from slices cut from the 3D model. In addition, the mechanical slicing process requires the original model to be destroyed. A careful experimental planning process should then be guided by FE simulations in order to determine the proper loading conditions and speed up the whole measurement process.

The purpose of the present paper is to describe a CAE approach, which combines FE simulations and photoelastic investigations for the stress analysis of complex 3D prototypes made by stereolithography. CAD geometries, used to manufacture RP models, are directly used to perform FE computations, thus allowing numerical simulations to become simpler and easier. In this paper, the photoelastic stress analysis of gear drives (spur gears and face gears) was carried out by exploiting models made of an epoxy-based resin through the stress-freezing technique. The face gear transmission, typically used in high performance aerospace drive systems, consists of a driving spur involute pinion and one or two conjugated face gears. The lack of published design experience and design standards, the complex geometries and unconventional operating conditions have led to the application of numerical simulations, mainly using finite element codes, for the analysis of their performances [8]. Such an analysis involves multiple contacts, edge contacts, non linearity and high stress gradient areas and therefore it is a good example of how experimental data can help to integrate numerical results obtained during the design process. In particular, the present work is aimed at highlighting potentialities and criticalities of the photoelastic analysis of complex 3D models guided by a FEM approach.

\section{Materials and Methods}

Figure 1 schematizes the entire process of manufacturing and experimental testing of 3D complex stereolithographic gear models adopted in the present work. Stereolithography was used to manufacture the models, geometrically defined by CAD. A simple but effective test apparatus was set-up with the aim of loading gear samples and FE analyses were used to support and plan the whole experimental activity. The experimental stress analysis was carried out by exploiting the principles of 3D photoelasticity which require stress freezing and subsequent slicing of the resin models. Results accomplished by numerical simulations have been also used as comparison to assess those experimentally obtained. 


\subsection{Digital photoelasticity}

Photoelasticity enables experimental stress analysis of two- and three-dimensional components to be performed by extracting information from two groups of interference fringe patterns emerging from a polariscope: isochromatics, which represent the contours of principal stress differences, and isoclinics, which are related to principal stress directions. Isoclinic and isochromatic fringe analysis yields two parameters for each point of the test piece:

1) the relative retardation $\delta$ (known also as fringe order) introduced by the photoelastic material between two polarized light beams transmitted through it, expressed in multiples of its wavelength. The relative retardation is proportional to the difference of principal stresses and is given, for a plane-stress state, by the relation:

$$
\delta=\frac{d \cdot\left(\sigma_{1}-\sigma_{2}\right)}{f_{\sigma}}
$$

where $\sigma_{1}$ and $\sigma_{2}\left(\sigma_{1} \geq \sigma_{2}\right)$ are the principal stresses, $d$ is the model thickness and $f_{\sigma}$ is the stress-optic coefficient for the wavelength of the light source used.

2) The angle of orientation, $\alpha$, between the maximum principal stress $\sigma_{l}$ and the horizontal reference axis.

When 3D photoelastic analyses are carried out, the models can be stress frozen and then mechanically sliced in order to evaluate the stress field. In particular, the model is loaded and allowed to go through a thermal cycling process known as stress freezing. At the end of the process, when the loads are removed, the model retains the stress field induced by the applied loads due to the bi-phase chemical structure of the resin [1]. Proper thin slices are then cut from the model and analyzed by using the principles of 2D photoelasticity. The slices retain the same stress distribution of the 3D model, provided the slicing operation is carried out carefully so that no machining stresses are introduced. This restriction is usually accomplished by using single-point cutting tools and providing appropriate coolant while cutting. 
A large class of problems, such as determination of stress concentration factors, stress intensity factors and contact stress parameters, require only the information of isochromatic fringe order [1]. In this work, among the various procedures proposed in literature in order to recover the isochromatic parameter [2], the photoelastic analysis of the fringe patterns was done by using the RGB method as proposed by [9]. RGB photoelasticity can give the total fringe order from a single color isochromatic full-field image obtained by using a white light source. This characteristic turns useful not only for the analysis of time varying phenomena but also for recovering small fringe orders from stress frozen slices.

\subsubsection{RGB method}

In RGB photoelasticity, the model is observed by a circular polariscope (Figure 2), generally configured in dark field. The isochromatics are acquired in white light by a color camera and decomposed into three primary colors, red, green and blue, by means of three wide band filters. The three levels of intensities, $R, G$ and $\mathrm{B}$, are digitized and used to perform full-field automatic analyses of isochromatics through comparison with calibrated values stored in a Look-Up-Table (LUT). The methodology is based on a calibration procedure which exploits a known stress field. The procedure consists of acquiring RGB triplets at each pixel along a transverse symmetry section of a calibration bending beam and storing the intensity values into an array (LUT). The RGB triplets correspond to retardations increasing linearly from a point where the retardation is zero to a point subjected to a maximum known retardation. In general, the material of the calibration specimen must match the material used in the test in order to take into account the dispersion of birefringence. If the first pixel of the calibration specimen corresponds to a zero fringe order (retardation $\delta_{0}=$ 0 ), the retardation $\delta_{i}$ corresponding to pixel $i$, increasing with retardation, is given by:

$$
\delta_{i}=N_{m} \frac{i}{i_{m}}
$$

where $N_{m}$ is the maximum relative retardation and $i_{m}$ is the number of the acquired RGB triplets calibrating the system. The R, G and B values in the LUT are directly linked with the retardation in the bending beam using monochromatic light. During the photoelastic analysis, the levels $R_{m}, G_{m}$ and $B_{m}$ are measured at those pixels in the specimen where the retardation has to be determined. Using the calibration array, the retardation corresponding to each set of $R_{m}, G_{m}$ and $B_{m}$ levels can be evaluated. The general procedure consists of [9]:

1) comparing the $R_{m}, G_{m}$ and $B_{m}$ values digitized at each point of the specimen with the $R_{i}, G_{i}$ and $B_{i}$ values stored in the LUT by means of the error function defined as:

$$
E_{i}=\left(R_{i}-R_{m}\right)^{2}+\left(G_{i}-G_{m}\right)^{2}+\left(B_{i}-B_{m}\right)^{2}
$$

for each index $i$ of the LUT;

2) searching the value of the index $i$ that minimizes the error function (3);

3) evaluating $\delta_{i}$ by means of equation (2).

Due to the attenuation of the RGB levels for retardations higher than 4 orders, the maximum measurable retardation is limited to three orders if incandescent lamps are used [9]. In this case, the retardation search procedure provides exact values with the exception of a few pixels where a measurement error can occur due to the similarity of colors of different orders. In cases where the light source is a fluorescent lamp having a 
discrete emission spectrum, it would be possible to determine higher fringe orders (up to 12) as reported in

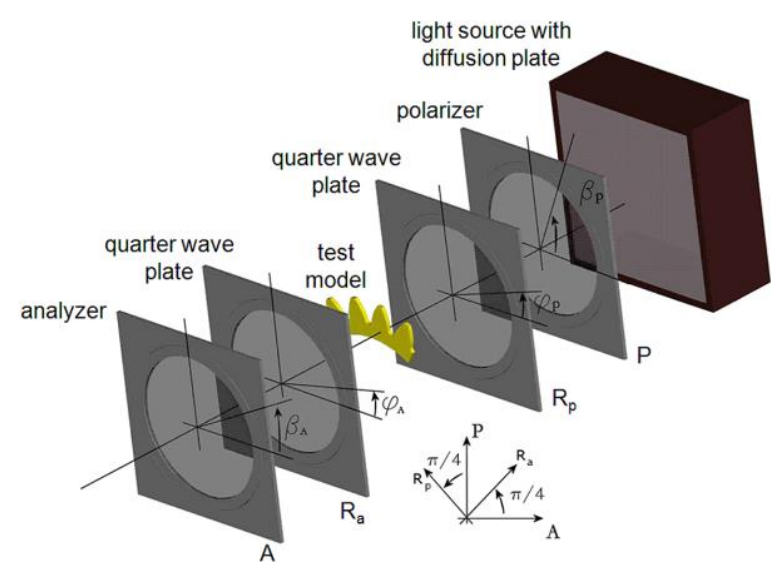

Fig. 2 Arrangement of the circular polariscope used for the RGB technique

\subsection{Photoelastic stress analysis for stereolithographic gear models}

The analysis of two different meshing conditions was carried out in order to test the developed framework. Firstly, the meshing of a pair of involute spur gears was considered in order to tune the procedure on a simple, known case. Then, the meshing of a face gear drive, consisting of a driving spur involute pinion and one conjugated face gear, was analyzed.

\subsubsection{Geometric models}

Three-dimensional photoelastic gear models were directly manufactured through a stereolithographic technique by using the StL models obtained from CAD models (Figure 3), thus reducing time and costs. While involute spur gears and pinions have a profile simple to define, face gear modeling involves the description of highly complex surface geometry. In this work, solid modeling of gear flank surfaces was done by interpolating data obtained from an enveloping generation code [11]. The face gear and the mating spur pinion were both modeled with the commercially available solid modeler Pro/Engineer. Points from the generation code were imported in an ASCII file as a set of ordered points belonging to contact lines of the shaper and the face gear. These points were interpolated with NURBS surfaces approximating the flank and the fillet of the face gear [8].

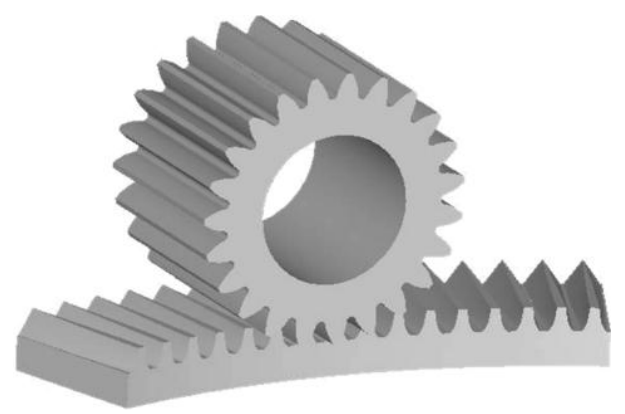

Fig. 3 CAD model of the face gear drive used for the stress field analysis

\subsubsection{Stereolithographic models}

All the models used in this work (Figure 4) were manufactured by stereolithography using the 3DSystems stereolithographic apparatus SLA $250 / 50$ and an epoxy resin, CIBATOOL® SL5170, which is photoelastically sensitive. In the current work the minimum possible layer thickness of $0.1 \mathrm{~mm}$ was used 
with the ACES (Accurate Clean Epoxy Solid) build style which produces extremely accurate, optically clear

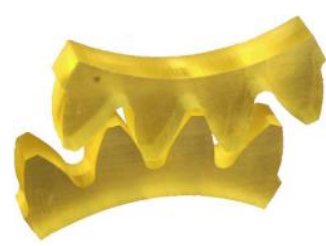

(a)

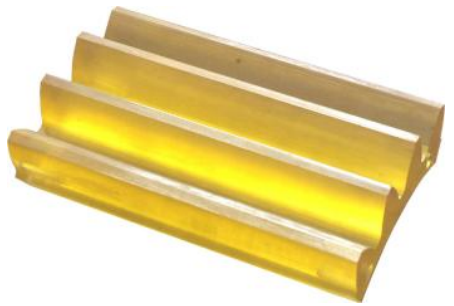

(b)

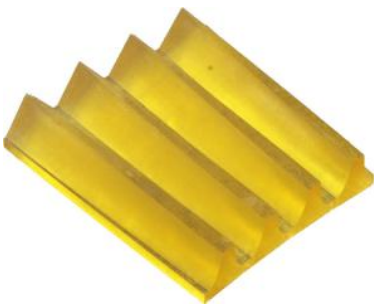

(c)

Fig. 4 Stereolithographic models used for the experimental analyses. (a) meshing of involute spur gears, (b,c) spur involute pinion and relative face gear sector, respectively

\subsubsection{Experimental set-up}

The experimental apparatus (Figure 5-a), designed to apply the load to the meshing gears, is very simple and small since it has to be housed in an oven. Figure 6 shows two photos of the apparatus in the oven with two meshing spur gear sectors (Figure 6-a) and the pinion and face gear sectors (Figure 6-b). It consists of a reference plate $(420 \times 300 \times 20 \mathrm{~mm})$ used to fix the face gear sector and of a horizontal drum $(250 \mathrm{~mm}$ diameter) to refer the spur involute pinion. The drum is free to rotate around its axis and to move along its vertical position thus allowing the relative distance of the gear pairs to be adjusted.

While the pinion sector is fixed to the flanges of the drum by means of threaded bolts, the reference features and fixing conditions to the plate are different in the cases of spur gear and face gear. The spur gear is fixed and referenced to the plate by means of an interface made of a $\mathrm{U}$-shaped profile provided with 4 slotted holes, for fixing the sector by means of bolts in different meshing positions (Figure 6-a).The face gear sector is rather fixed and referenced directly to the plate by means of aluminum profile bars (as shown in Figure 5b) and by a pin defining its radial placement. The torque is simply applied by calibrated weights hanged directly to the flanges of the drum or to cantilever beams fixed to the flanges in order to increase to load arm (Figure 6-b). 


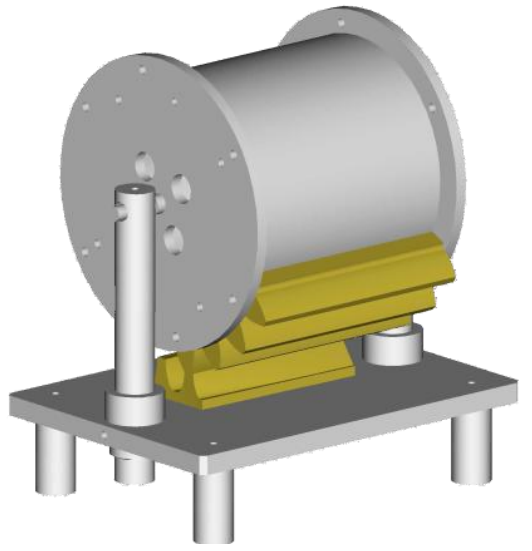

(a)

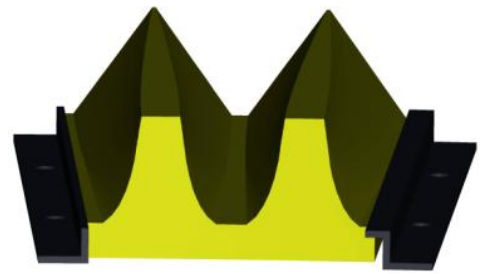

(b)

Fig. 5 (a) CAD model of the experimental apparatus, (b) aluminum profile bars used to fix the face gear sector to the reference plate

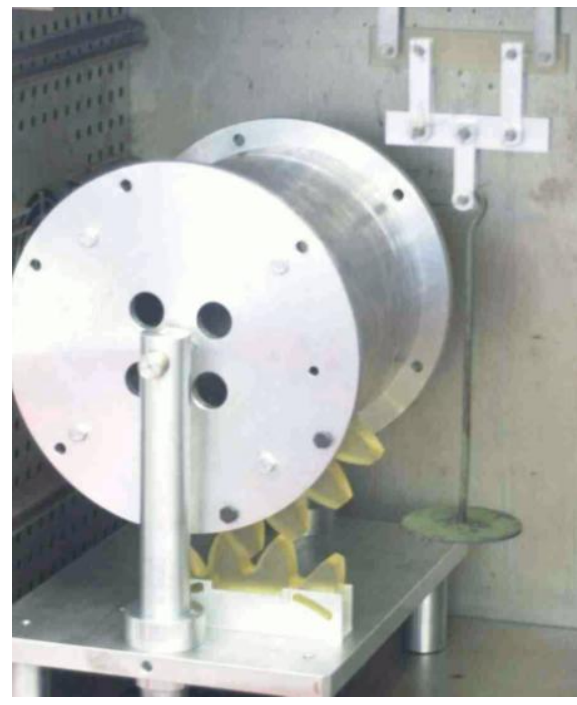

(a)

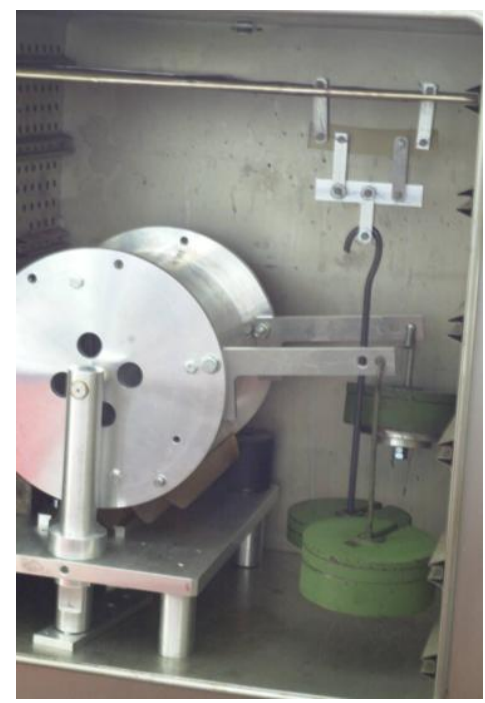

(b)

Fig. 6 Pictures of the experimental apparatus housed inside the oven: (a) involute spur gear meshing, (b) meshing of a face gear drive

The drum is hollow and some holes were realized in the flanges with the aim of avoiding excessive heat accumulation in the model areas in contact with the apparatus, thus preventing thermal distortions. Figures 6 also show the placement of calibration specimen, along with its four-point-bending setup, inside the oven. In order to avoid erroneous evaluations of the fringe order through the RGB method, the prismatic calibration specimen undergoes the same stress freezing treatment of the test gear drives. This condition also allows a reliable and customized computation of the stress-optic material coefficient to be used for photoelastic analyses. The images acquisition process was performed by using the digital photocamera Nikon ${ }^{\circledR} \mathrm{D} 1$ having a resolution of $1300 \times 2000$ pixels for 16 millions of colors. The apparatus used for the thermal cycle is a forced air-heating oven equipped with a probe PT100 for temperature control and governed by the automatic controller ASCON XS3001/CAA/99999999. 


\subsubsection{Stress freezing and model slicing process}

Three-dimensional photoelasticity using the stress-freezing technique is dependent on resin models that do not possess any residual stress due to the manufacturing process. For this reason, in order to remove the residual stresses in the prototypes caused by the post-curing resin shrinkage, all the samples were annealed at resin glass transition temperature $\left(T_{g}\right)$ before experimental tests. The stress-freezing cycle was divided into four steps (Figure 7):

1) A linear temperature raise from room temperature to a temperature slightly below $T_{g}$. A rate of $4{ }^{\circ} \mathrm{C} / \mathrm{h}$ was used.

2) A constant temperature interval (from 5 to 10 hours) to assure heat uniformity throughout the models. The interval width was determined accordingly to the size of the prototypes used.

3) A first cooling rate $\left(2{ }^{\circ} \mathrm{C} / \mathrm{h}\right)$ down to $65^{\circ} \mathrm{C}$.

4) A second higher cooling rate $\left(3{ }^{\circ} \mathrm{C} / \mathrm{h}\right)$ down to room temperature. Once the critical stress freezing temperature $\left(T_{c}\right)$ is reached, secondary bonds are re-formed between the elastically deformed primary bonds. For this reason, a slightly faster cooling rate can be used in order to speed up the thermal cycle.

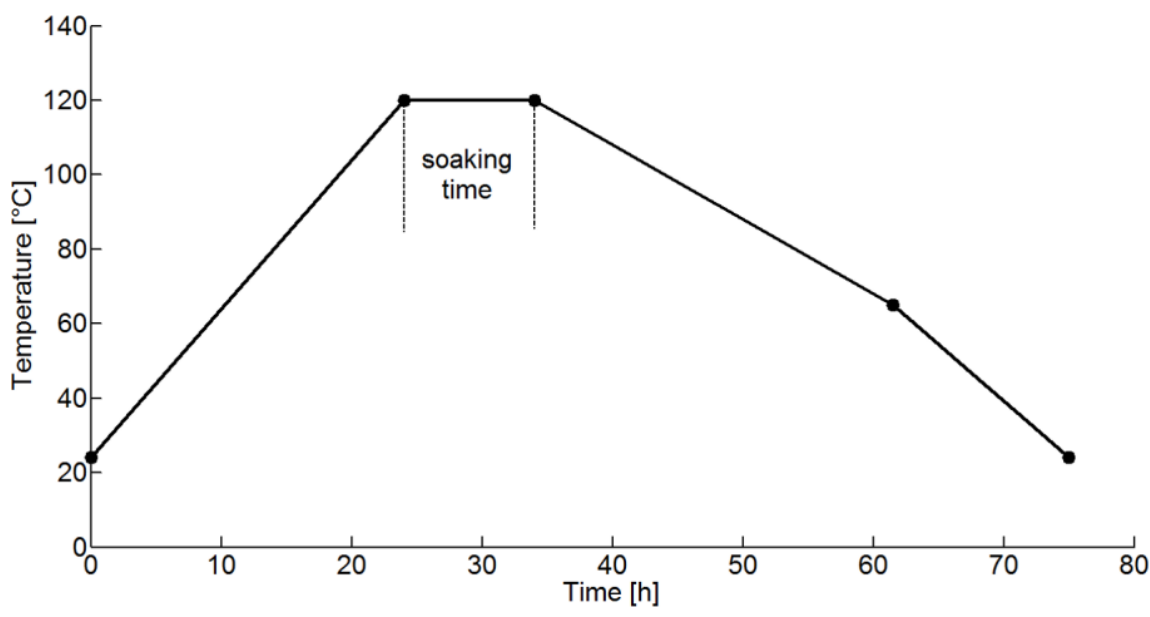

Fig. 7 Stress-freezing thermal cycle adopted for the experimental tests

Even if loads are usually not applied to the test models until the stress-freezing temperature is reached, in this work the loads were applied before the beginning of the stress-freezing and kept throughout the complete thermal cycle. This choice was adopted in order to avoid possible thermal shocks.

Model slicing was then performed in order to carry out a direct transmission photoelastic analysis. If the slice does not correspond to a principal plane, the stresses in the slice plane are secondary principal stresses. Since it is not always possible to identify the principal planes, the slicing was carried out through planes parallel to the free surfaces of the three-dimensional models. The slice thickness must be determined to accomplish two opposite requirements: 1) sufficiently thin with respect to the model size in order to ensure that the stresses do not change in either magnitude or direction through the slice thickness, 2) sufficiently thick to assure a minimal number of fringe orders for an accurate photoelastic analysis.

Slices having constant thickness (Figure 8) were then obtained by using a milling machine with a $2 \mathrm{~mm}$ thick cutter, rotating at high speed $(1500 \mathrm{rpm})$. The cutter must be cooled with a large amount of coolant to prevent the model overheating, which would result in distortion or annealing of the fringe pattern. 


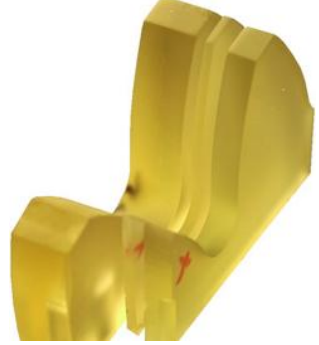

(a)

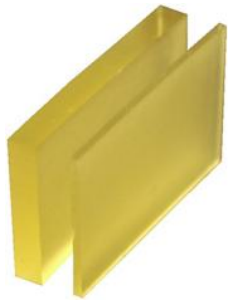

(b)

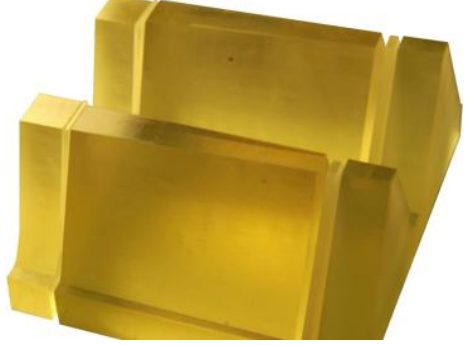

(c)

Fig. 8 Slicing process regarding involute spur gear (a), calibration specimen (b) and face gear sector (c) respectively

After the slicing process, each slice was manually finished by using 500-grit sandpaper. Moreover, to further augment their transparency and the optical clarity of the fringe patterns, the slices were coated by a film of mineral oil.

\subsubsection{Calibration of the model material}

A prismatic specimen, in a four-point-bending set-up (Figure 9-a), was used to calibrate the photoelastic material properties (stress-optic coefficient) at $T_{g}$ [15]. If $l_{2}$ is the loading span and $\left(l_{2}+2 l_{l}\right)$ the support span, then in the central portion, at a distance of one beam thickness from the loading points, the stress state is uniaxial and can be written as:

$$
\sigma_{x}(y)=\frac{6 \cdot P \cdot l_{1}}{d \cdot h^{3}} \cdot y
$$

where $P$ is the total applied load. From equation (1), considering $\sigma_{2}=0$, it derives that:

$$
f_{\sigma}=\frac{6 \cdot P \cdot l_{1}}{h^{3}} \cdot \frac{|y|}{\delta(y)}
$$

The fringe order varies linearly from 0 , on the neutral axis, to its maximum values on lower and upper extremities of the specimen. A plot relating integer values of the fringe order, extracted from isochromatics as observed with a circular dark-field polariscope (Figure 9-b), with the distance from the inferior edge of the specimen can be obtained (Figure 10). A linear regression is then performed by using the integer fringe orders in the central region of the specimen and used to extrapolate the fringe orders $N_{i}$ and $N_{s}$ on the inferior and superior specimen edges, respectively. The stress-optic coefficient can then be computed by using relation (5) as:

$$
f_{\sigma}=\frac{6 \cdot P \cdot l_{1}}{h^{3}} \cdot \frac{h}{N_{i}+N_{s}}
$$

Given $h=30 \mathrm{~mm}, l_{l}=25 \mathrm{~mm}, d=11 \mathrm{~mm}, l=150 \mathrm{~mm}$ and $\mathbf{P}=45 \mathrm{~N}$, a mean value for the stress-optic coefficient $f_{\sigma}=0.82 \mathrm{~N} / \mathrm{mm} /$ fringe was determined. 


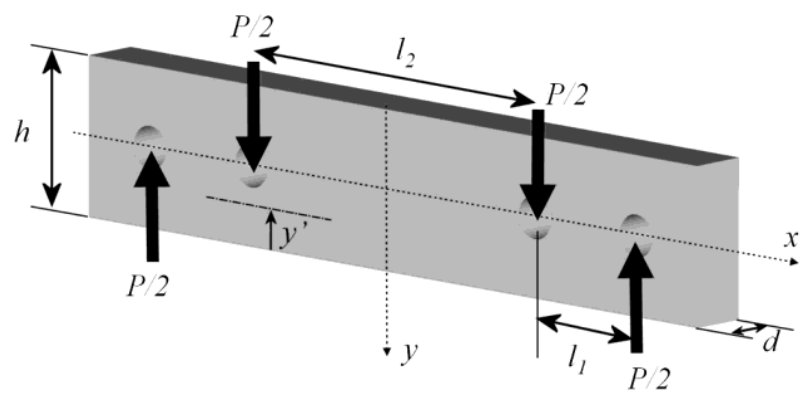

(a)

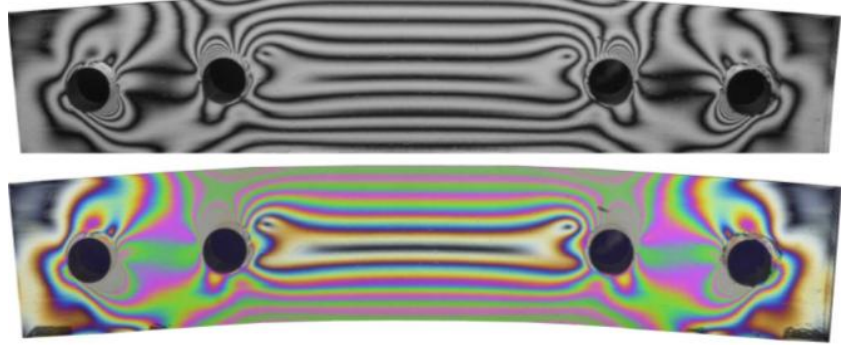

(b)

Fig. 9 (a) Prismatic specimen in a four-point-bending set-up used for the calibration of the material properties, (b) isocromatics emerging from a dark field polariscope (in monochromatic and white light) after a stress-freezing cycle

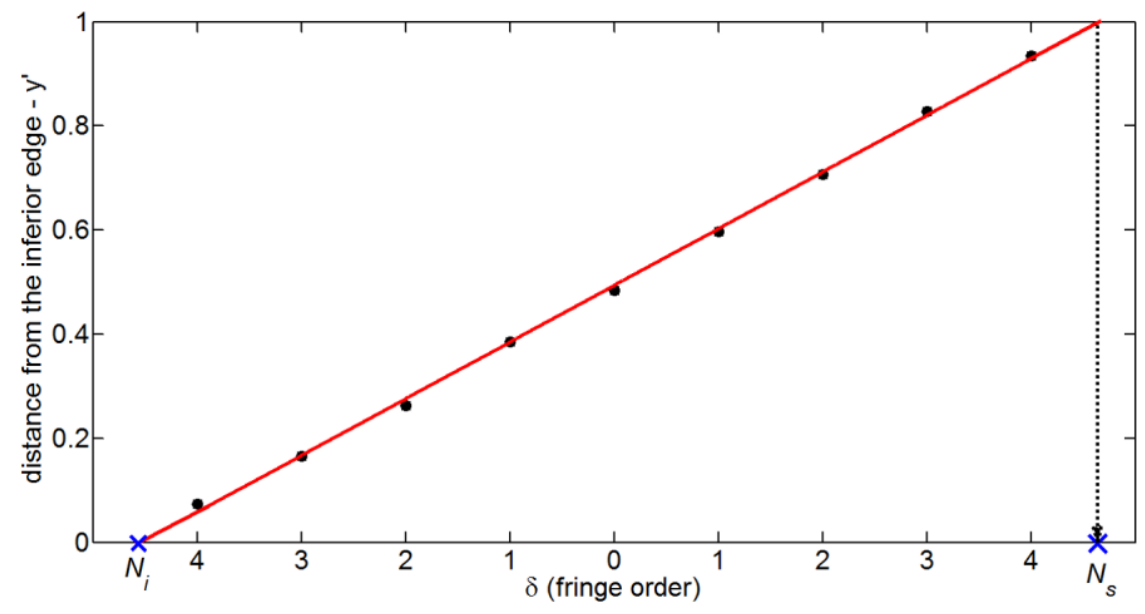

Fig. 10 Fringe order plot with respect to the distance from the inferior specimen edge

The resin Elastic Modulus at $T_{g}$ must also be determined in order to carry out also a finite element analyses. The elastic deformation induced in the prismatic specimen at the stress-freezing temperature becomes permanent when it returns to room temperature (Figure 9-b) and can be used to determine the resin elastic modulus at the stress-freezing temperature [15]. A mean value for the elastic modulus $E=18 \mathrm{MPa}$ was thus determined through experimental tests.

\subsubsection{Finite element analysis}

The finite element investigation was carried out in order to define the loading set-up for the 3D photoelastic analysis. Loading conditions must be accurately estimated since mechanical slicing destroys the model and only a single loading case can be considered for each model.

Tooth modeling and complete gear solid modeling, as well as the relative placement of the gear pair in the meshing cycle, was performed with Pro/Engineer. The CAD models were imported into the FEA environment by the IGES standard data exchange to obtain a better control on mesh generation. Mesh generation, pre-processing, and FE analysis were accomplished by Ansys ${ }^{\circledR}$. Meshing simulation improves considerably if a regular mesh is used on contact surfaces. This was achieved by using isoparametric brick elements with 8 nodes and generating a volumetric solid mesh by sweeping the elements created on the tooth front surface. Contact and target elements, necessary to build the contacting pair FE model, were then 
defined to simulate gear meshing condition. Whole toothed sectors were finally modeled using coarser meshes for the unloaded teeth (Figure 11). This allows for the numerical model to be as similar as possible to the experimental one.

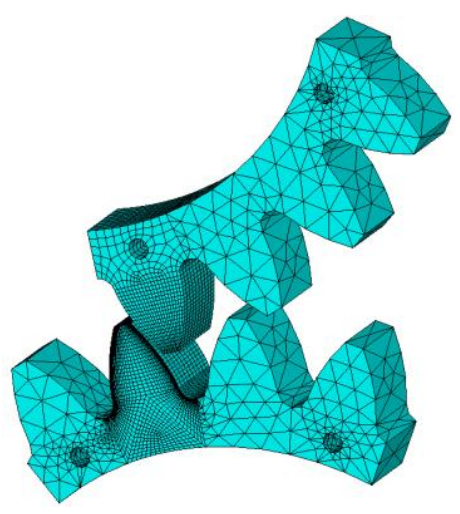

(a)

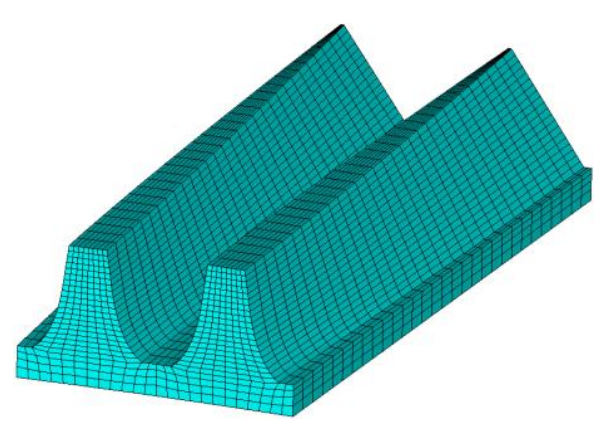

(b)

Fig. 11 Meshed involute spur gears (a) and face gear sector (b)

The spur gear sector model was constrained in correspondence of its holes (Figure 12-a) while the face gear sector was constrained on the reference plate and in correspondence of aluminum profile bars (Figure 12-b). The rotation of the pinion sector was obtained by defining a master node on the pinion axis, free only to rotate, and linking it to slave nodes on the bolt holes through constraint equations (Figure 12-c). The loading conditions is simulated by applying a torque around the rotation axis.

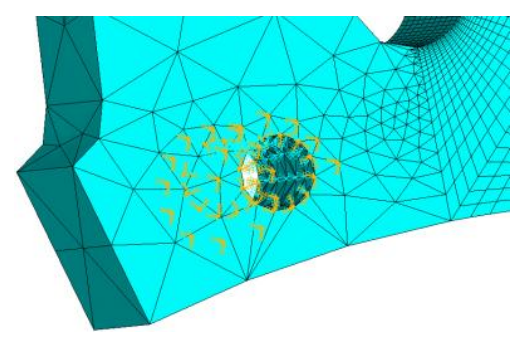

(a)

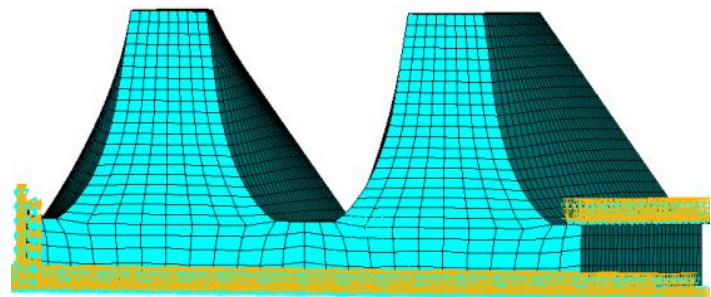

(b)

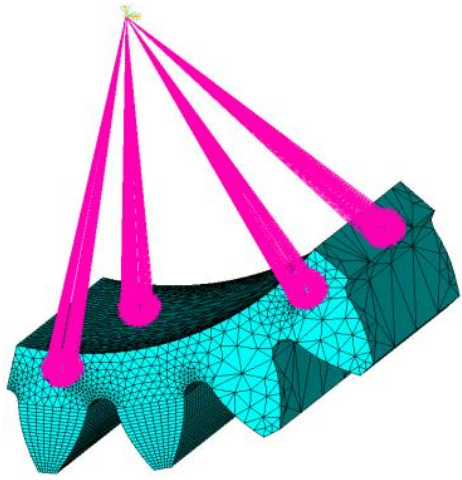

(c)

Fig. 12 Constraints applied to the involute spur sector (a) and face gear sector (b). (c) Master and slave nodes used to constrain the pinion sector to rotate around its axis 
Particular care was taken in dealing with the initial contact conditions because it could cause convergence problems due to rigid body motion or to highly non-linear contact load. In simulations where rigid body motions are constrained only by the presence of contact condition, contacting bodies should be modeled in 'just touching' position to generate a numerically stable problem and the torque applied gradually, increasing it by steps. The material properties were defined accordingly to those of the resin at the stress-freezing temperature, obtained by the calibration procedures $(E=18 \mathrm{Mpa}, v=0.48)$. Moreover, since the experimental tests are carried out in the oven, temperature and its effects were considered in simulations through a thermal expansion coefficient.

Figure 13 shows an example of principal stresses difference maps obtained by the FEM simulations. For the spur gear pair the applied torque to the pinion was $9960 \mathrm{~N} \cdot \mathrm{mm}$, for the face gear pinion $5 \cdot 10^{4} \mathrm{~N} \cdot \mathrm{mm}$. The FEM models were also sectioned correspondingly to the slices obtained from the resin models and the numerical results in terms of principal stress difference distribution were compared to the experimental ones, as will be shown in the following section.

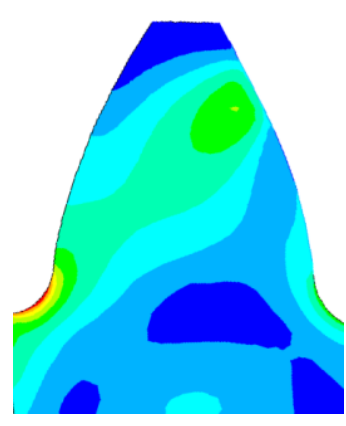

(a)

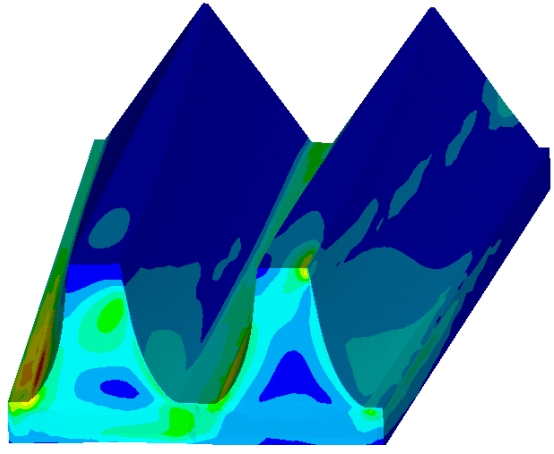

(b)

Fig. 13 FEM principal stress difference maps for spur (a) and face gear (b) sectors

\section{Results}

Figure 14 shows the isochromatic patterns in white light, as observed from slices cut within the spur gear pair, by using a circular polariscope in the dark field configuration. The results are relative to a $1.5 \mathrm{~mm}$ (Figure 14-a), $2 \mathrm{~mm}$ (Figure 14-b) and $3 \mathrm{~mm}$ (Figure 14-c) thick slices. The higher fringe order observed in the thicker slice is due to the linear dependence of the retardation with respect to the model thickness.

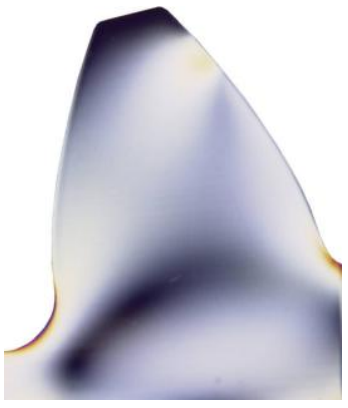

(a)

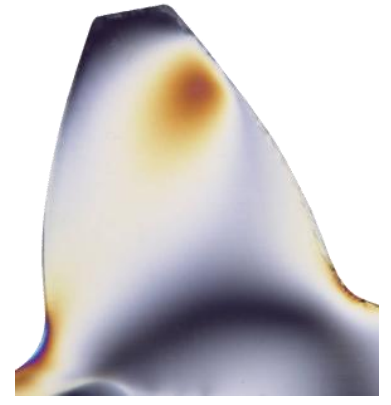

(b)

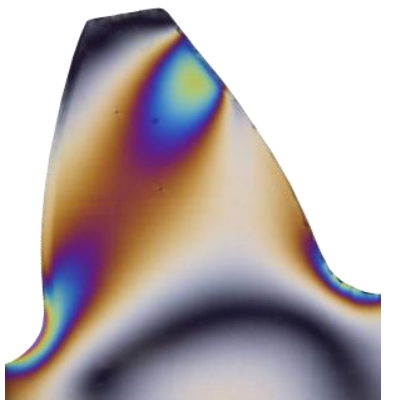

(c)

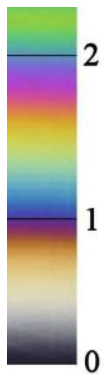

(d)

Fig. 14 Dark-field isochromatic fringes in white light observed for a $1.5 \mathrm{~mm}$ (a), $2 \mathrm{~mm}$ (b) and $3 \mathrm{~mm}$ (c) thick slices. (d) calibration array used to create the LUT for RGB photoelasticity and derived from a slice of the same specimen used for the stress-optic calibration 
The calibration array used for RGB photoelasticity was derived from the isochromatics observed in the same specimen used for the stress-optical calibration and the integer fringe orders are obtained by using monochromatic light. The load to be applied to the calibration specimen must be evaluated with particular attention: the fringes of the model can be compared to those of the calibration specimen only if the light intensities are the same. It is therefore necessary to obtain slices from the calibration specimen with thickness comparable to those to be analyzed also containing the same fringe order to make the study of the whole slice possible. Figure 15 reports, as example, the full-field maps of the retardation obtained by processing, with the RGB method, the images shown in Figure 14-b and Figure 14-c, respectively.

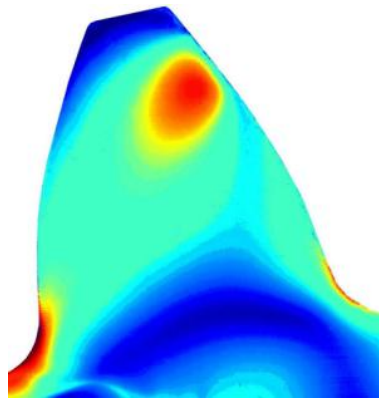

(a)

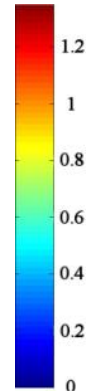
22

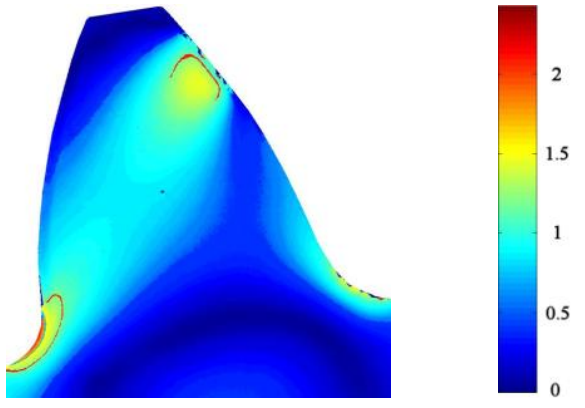

(b)

Fig. 15 Full-field maps of the retardation obtained by processing the images shown in Figure 14-b (a) and Figur14-c (b), by using the RGB method

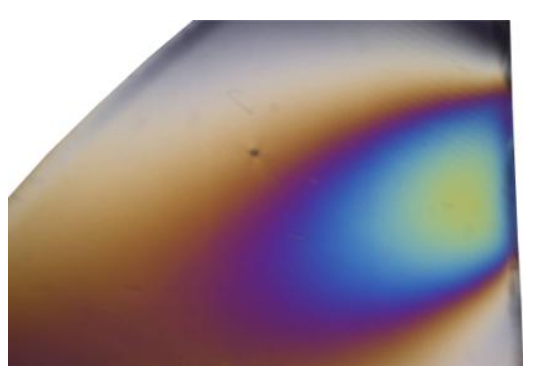

(a)

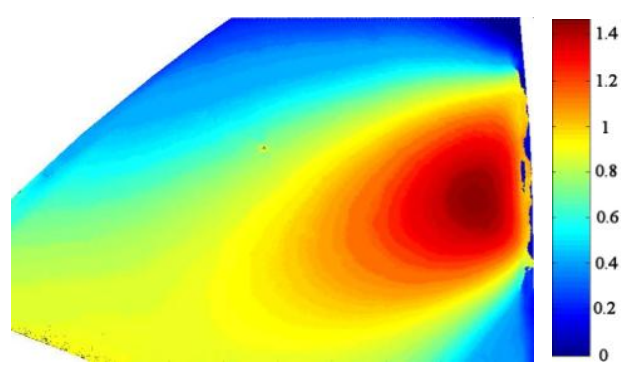

(c)

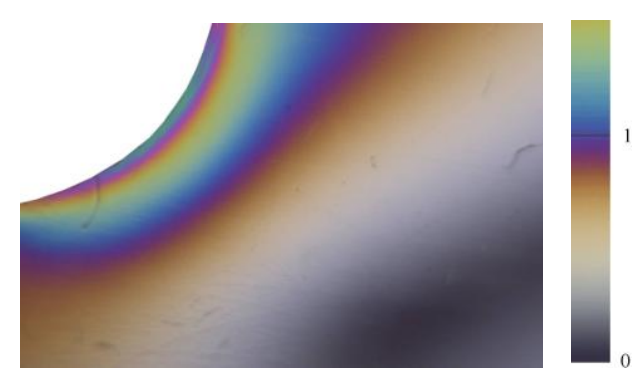

(b)

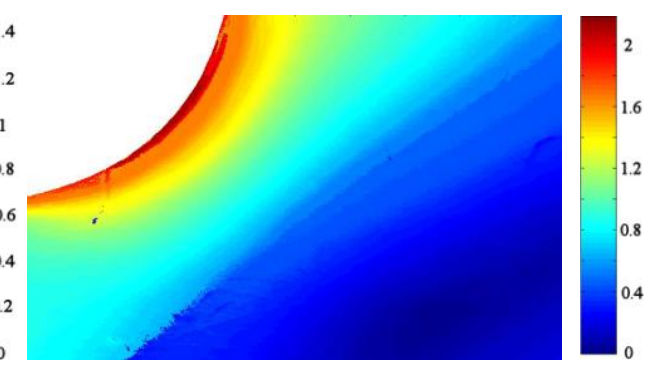

(d)

Fig. 16 Isochromatics in white light relative to the contact area (a) and to the root of the tooth (b) in the 3 $\mathrm{mm}$ thick slice along with the respective retardation maps obtained with the RGB method (c,d)

Since of the high difference of the fringe gradient in the model from that in the calibration specimen in the areas of stress concentration, the assignment of the right value of the retardation can be difficult. That can bring the rise of spots on the maps of the retardation (as shown in Figure 15-b) in boundary areas that are 
themselves more subjected to disturbances. To solve this problem the image of these areas can be magnified (Figures 16) to make the image fringe gradient not higher than 0.1 order/pixel.

Figures 17 present the comparison between numerical and the experimental results along the contact line (Figure 17-a) and along the direction normal to the free edge of the tensile side of the gear tooth fillet (Figure 17-b). The curve of the experimental results is referred to data obtained by the RGB method by processing the $2 \mathrm{~mm}$ thick slice.

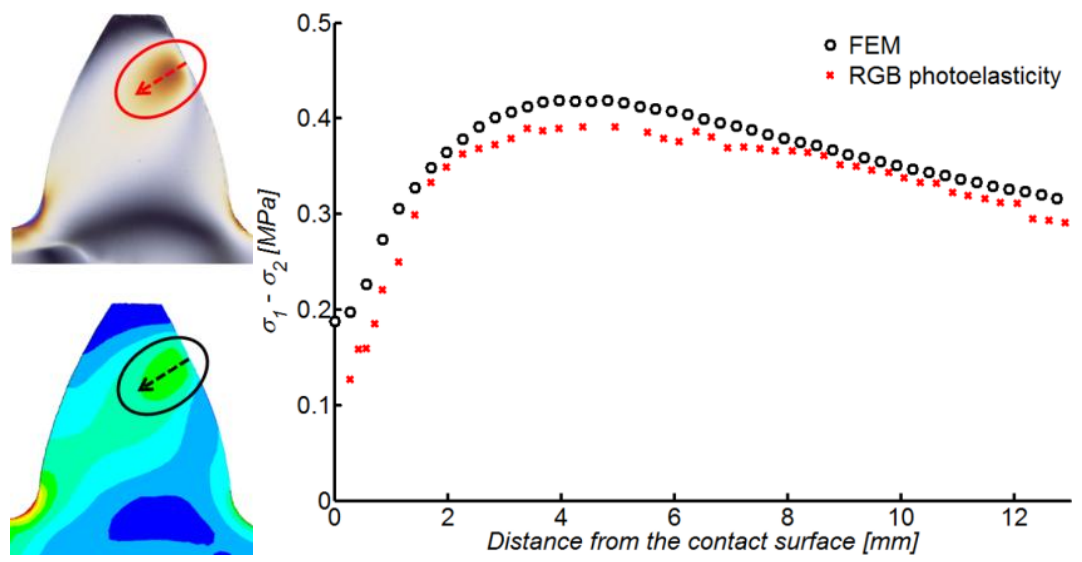

(a)
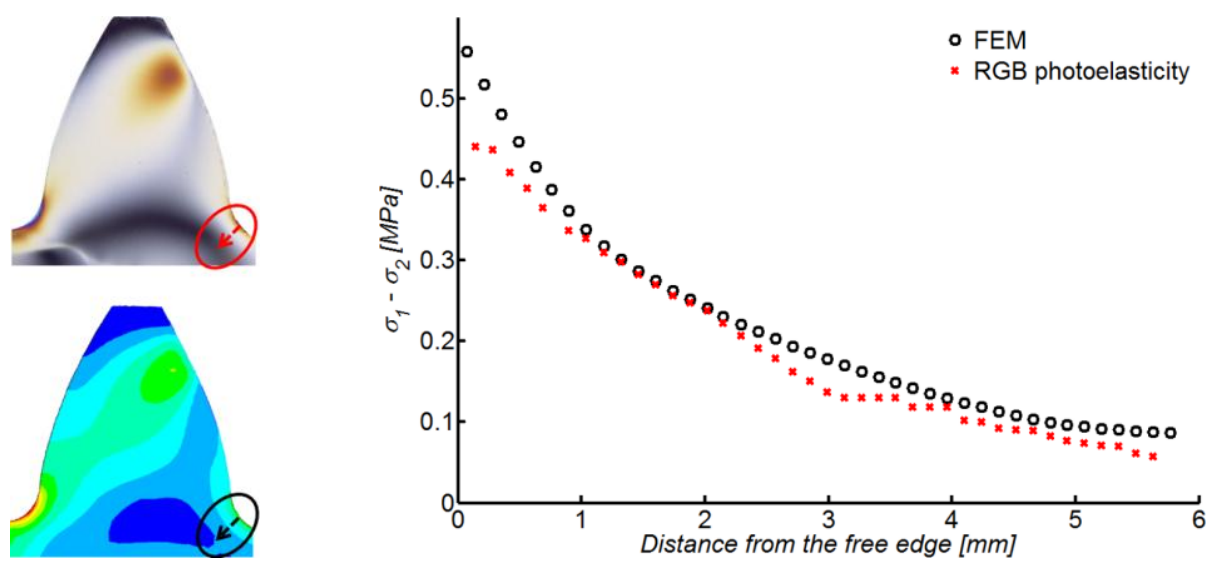

(b)

Fig. 17 Numerical and experimental results of the difference of the principal stresses along the contact line (a) and the direction normal to the free edge of the tensile side of the gear tooth fillet (b). The experimental results have been calculated by using the RGB method for the $2 \mathrm{~mm}$ thick slice

Except for the boundary areas where a certain data dispersion can be observed, the agreement of the two curves is quite satisfactory. The experimental curve stays below the numerical one. This circumstance was almost expected since the difference of the principal stresses observed experimentally is, actually, averaged through the thickness of the slice, and an interpolation is made.

Finally, the experimental test involving face gear drives was carried out by simulating the meshing of pinion and gear sectors with two teeth. Because of the resin large strain value, the contact area results quite large on both teeth (as can be seen in Figure 18-b), causing an excessive load distribution that hinders a 3-D photoelastic analysis. Therefore the minimum torque to be applied in order to visualize isochromatic fringes in slices not thicker than $2 \mathrm{~mm}$ was determined by a preliminary finite element analysis. $1.8 \mathrm{~mm}$ thick slices 
were then extracted along the whole longitudinal dimension of the face gear to verify the meshing of the two teeth (Figure 18-a).

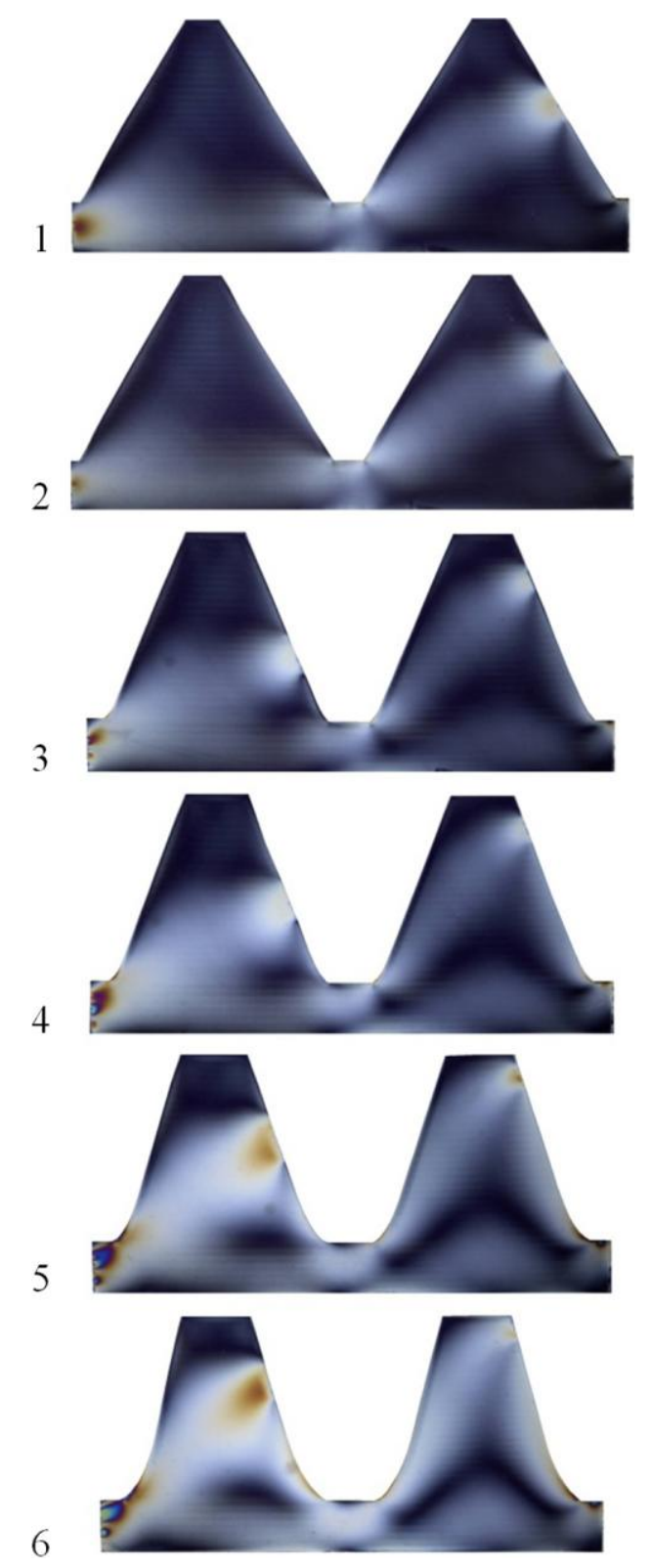

(a)

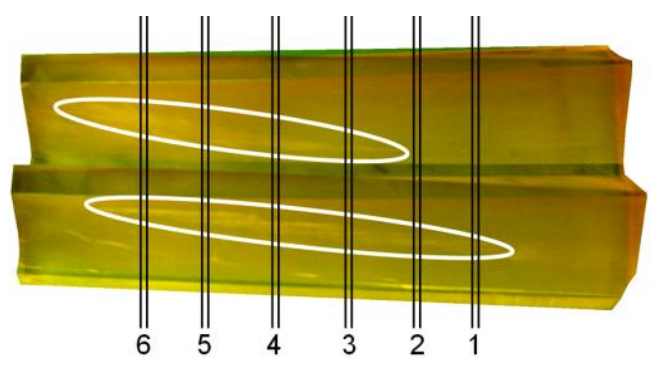

(b)

Fig. 18 (a) Dark-field isochromatic fringes in white light observed for $1.8 \mathrm{~mm}$ thick slices obtained along the whole longitudinal dimension of the teeth, (b) contact area visualized on the face gear flank surfaces

In Figure 19 some examples of retardation values determined by using the RGB photoelasticity along with a comparison with the results obtained with FEM are reported. Differences are due to the experimental gear position error, unavoidable and not easily measurable with the used fixture. 

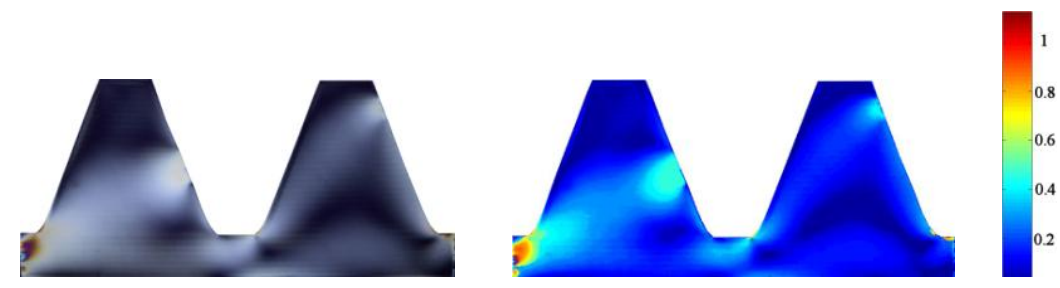

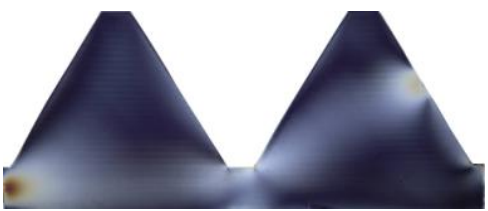

(a)

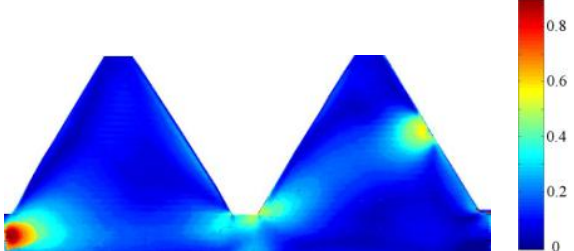

(b)
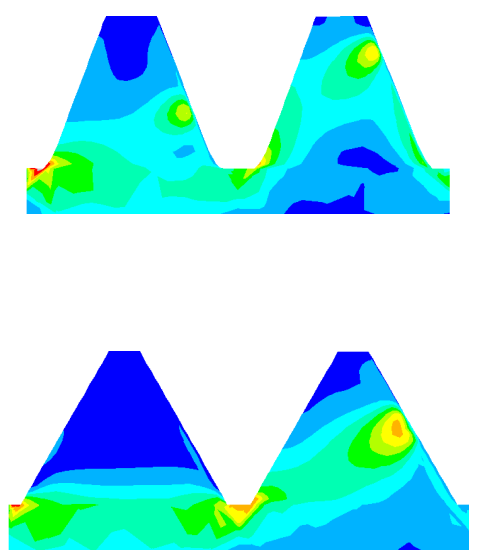

(c)

Fig. 19 (a) Dark-field isochromatic fringes and (b) relative retardation maps obtained with the RGB method for a $1.8 \mathrm{~mm}$ thick slice. (c) Difference of the principal stresses obtained by the finite element analysis

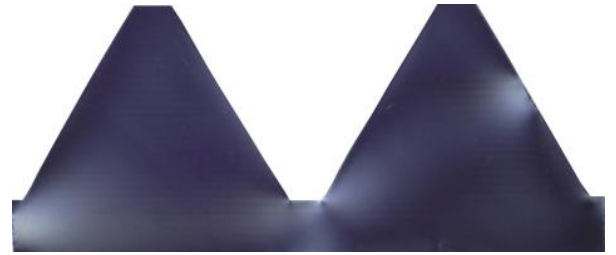

(a)

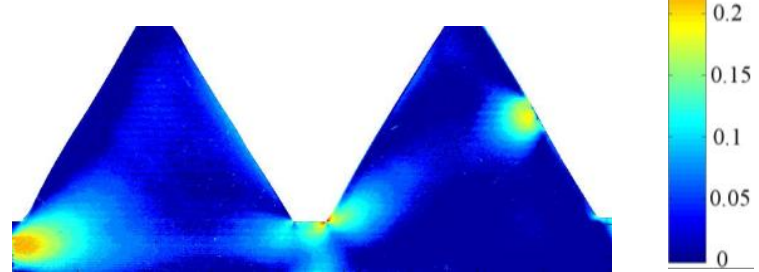

(b)

Fig. 20 (a) Example of dark-field isochromatic fringes for a $0.8 \mathrm{~mm}$ thick slice and (b) relative retardation map obtained with the RGB method

As demonstrated by Figure 20, the fringe order for a $0.8 \mathrm{~mm}$ slice seems to be too low for a reliable photoelastic analysis and a higher load combined with a resin having different mechanical properties, in particular higher stiffness to avoid excessive strain, should be used. In such cases, the limit of the used stereolithographic resin emerges as well as the usefulness of an accurate experimental planning carried out with proper numerical simulations.

\section{Discussion and conclusions}

In this paper, a CAE approach for the stress analysis of complex mechanical components was proposed by integrating FEM simulations with 3D photoelasticity. In particular, the stress field distribution of face gear models made by stereolithography was studied. Even if many are the parameters involved in the problem (misalignments in the gears meshing due to the loading apparatus, discrepancies between CAD geometries and real manufactured shapes, finite thickness of the slices used for photoelastic analyses), the obtained results show a substantial agreement between numerical simulations and experimental data. The main noticeable differences between numerical and photoelastic analyses can be ascribed to the experimental stress field average which is intrinsically caused by the finite slice thickness. Moreover, residual stresses introduced by manufacturing, and not completely annealed by the stress relieving process, affect the final 
stress field within the contact areas. Surface finishing properties, not accounted for in the ideal geometry, may further contribute to the differences between results especially in the proximity of the contact areas.

Despite the above described criticalities, the present study attests the potentialities of the adopted approach as well as the usefulness of such stereolithographic models to perform 3D photoelastic analyses guided by numerical simulations. Moreover, FE analyses demonstrated their usefulness to minimize the number of experiments and optimize the slicing process thus providing cost- and time-effective experimental photoelastic analyses.

It is worth mentioning that potential differences between CAD geometries and stereolithographic models have not been taken into account in the present work. However, real manufactured geometries could be easily considered by acquiring the resin prototypes with non-contact reverse engineering techniques, as, for example, a full-field optical scanner [16]. The direct use of the optically acquired shapes for FE simulations would produce numerical results which are based on the same geometry that originates those experimentally obtained. Moreover, this would allow the extension of the presented framework applicability for the analysis of handcrafted components when CAD models are not available.

Future works will investigate the framework applicability in new research fields, particularly where the use of RP technique is increasing. RP models have been traditionally used as complementary design tools. In recent times, however, there has been a growing interest in the use of RP techniques to produce real models which are adopted within many fields. For example, in biomedical applications (prostheses design, orthodontic treatments, surgical dental guides) there is an extensive use of customized prototypes, and the possibility to easily integrate numerical and experimental analysis could offer high potentialities for the optimization and validation of components design. Also in the field of industrial applications, there is a growing use of direct 3D printed models as actual components which could benefit from the presented framework (i.e. small plastic gearbox, generic customized components). The introduction of customized photoelastic analyses for real components would greatly enhance the detection of possible criticalities arising from challenging applications. The described framework, even if presented for a specific test case, can be easily extended to different applications without losing its effectiveness.

\section{References}

1. Ramesh, K.: Digital Photoelasticity: Advanced Techniques and Applications. Springer-Verlag New York, Inc., (2000)

2. Ramesh, K., Kasimayan, T., Simon, B.N.: Digital photoelasticity - A comprehensive review. J Strain Anal Eng 46(4), 245-266 (2011). doi:Doi 10.1177/0309324711401501

3. Bártolo, P.: Stereolithography materials, processes and applications. Springer, New York (2011)

4. Curtis, J.D., Hanna, S.D., Patterson, E.A., Taroni, M.: On the use of stereolithography for the manufacture of photoelastic models. Exp Mech 43(2), 148-162 (2003). doi:Doi 10.1177/0014485103043002005

5. Karalekas, D.E., Agelopoulos, A.: On the use of stereolithography built photoelastic models for stress analysis investigations. Mater Design 27(2), 100-106 (2006). doi:DOI 10.1016/j.matdes.2004.09.028

6. Galietti, U., Pappalettere, C., Quarta, V.: Photoelastic characterization of stereolithographic epoxy resins and study of a car engine bracket 3-D model. Paper presented at the Proc. of the 2002 SEM Annual Conf. on Experimental and Applied Mechanics, Milwaukee, Wisconsin, USA, 10-12 June 2002

7. Ashokan, K., Prasath, R.G.R., Ramesh, K.: Noise-Free Determination of Isochromatic Parameter of Stereolithography-Built Models. Exp Techniques 36(2), 70-75 (2012). doi:DOI 10.1111/j.17471567.2010.00695.x

8. Barone, S., Borgianni, L., Forte, P.: Evaluation of the effect of misalignment and profile modification in face gear drive by a finite element meshing simulation. J Mech Design 126(5), 916-924 (2004). doi:Doi 10.1115/1.1767818 
9. Ajovalasit, A., Barone, S., Petrucci, G.: Towards Rgb Photoelasticity - Full-Field Automated Photoelasticity in White-Light. Exp Mech 35(3), 193-200 (1995). doi:Doi 10.1007/Bf02319657

10. Ajovalasit, A., Petrucci, G., Scafidi, M.: RGB Photoelasticity: Review and Improvements. Strain 46(2), 137-147 (2010).

11. Barone, S.: Gear geometric design by B-spline curve fitting and sweep surface modelling. Eng Comput 17(1), 66-74 (2001). doi:DOI 10.1007/s003660170024

12. Madhu, K.R., Prasath, R.G.R., Ramesh, K.: Colour adaptation in three fringe photoelasticity. Exp Mech 47(2), 271-276 (2007). doi:DOI 10.1007/s11340-006-9012-x

13. Simon, B.N., Kasimayan, T., Ramesh, K.: The influence of ambient illumination on colour adaptation in three fringe photoelasticity. Opt Laser Eng 49(2), 258-264 (2011). doi:DOI 10.1016/j.optlaseng.2010.09.018

14. Simon, B.N., Ramesh, K.: Colour Adaptation in Three Fringe Photoelasticity Using a Single Image. Exp Techniques 35(5), 59-65 (2011). doi:DOI 10.1111/j.1747-1567.2010.00646.x

15. SPOTS: Standardisation Project for Optical Techniques of Strain Measurement. Draft SPOTS Standard Part III (5): Good Practice Guide to Reflection Photoelasticity. http://www.opticalstrain.org/spots_guides.htm (2006). Accessed 25/07/2013

16. Barone, S., Paoli, A., Razionale, A.V.: Multiple alignments of range maps by active stereo imaging and global marker framing. Opt Laser Eng 51(2), 116-127 (2013). doi:DOI 10.1016/j.optlaseng.2012.09.003 